\title{
Percepções atribuídas por enfermeiros sobre a relação entre a inovação e a
}

\section{enfermagem}

\author{
Perceptions assigned by nurses on the relationship between innovation and nursing \\ Percepciones asignadas por las enfermeras sobre la relación entre innovación y enfermeira
}

Recebido: 13/05/2021 | Revisado: 18/05/2021 | Aceito: 18/05/2021 | Publicado: 06/06/2021

\author{
Kauan Tamandaré Oliveira \\ ORCID: https://orcid.org/0000-0002-4253-4751 \\ A.C. Camargo Cancer Center, Brasi \\ E-mail: kauantamandare@hotmail.com \\ Luiza Watanabe Dal Ben \\ ORCID: https://orcid.org/0000-0001-7847-5602 \\ Dal Bem Home Care, Brasil \\ E-mail: luiza@dalben.com.br
}

\begin{abstract}
Resumo
Objetivo: Descrever as percepções de enfermeiros gestores sobre a relação da Enfermagem com a inovação no processo de trabalho. Método: Trata-se de um estudo quantitativo, descritivo, exploratório, realizado em um hospital especializado oncológico, de grande porte, de alta complexidade e filantrópico, localizado na região central da cidade de São Paulo. Resultados: Participaram 54 enfermeiros envolvidos no processo de trabalho administrar em enfermagem. Os entrevistados apresentaram alta percepção sobre enfermagem e inovação. A representação na inovação na enfermagem está vinculada a criação de materiais e equipamento. Discussão: A inovação é um assunto importante para o cenário de saúde e faz parte da gestão e do processo de trabalho, amadurecido através de ensino, educação continuada e vivência. Conclusões: A inovação, sem sombra de dúvida, proporciona o sucesso ao processo e ao sistema de saúde e, portanto, a ausência de um método de inovação influencia a falta de clareza no dia a dia. provoca a baixa ou não valorização do tema na prática.
\end{abstract}

Palavras-chave: Difusão de inovações; Prática profissional; Serviços de enfermagem; Administração em enfermagem; Enfermagem; Inovação.

\begin{abstract}
Objective: To describe the perceptions of nurse managers about the relationship between Nursing and innovation in the work process. Method: This is a quantitative, descriptive, exploratory study, carried out in a specialized oncology hospital, large, highly complex and philanthropic, located in the central region of the city of São Paulo-SP. Results: 54 nurses participated in the nursing administration work process. Respondents had a high perception of nursing and innovation. Representation in innovation in nursing is linked to the creation of materials and equipment. Discussion: Innovation is an important issue for the health scenario and is part of management and the work process, matured through teaching, continuing education and experience. Conclusions: Innovation, without a doubt, provides success to the health system and process and, therefore, the absence of an innovation method influences the lack of clarity in everyday life. causes the low or no appreciation of the theme in practice.
\end{abstract}

Keywords: Diffusion of innovations; Professional practice; Nursing services; Nursing administration; Nursing; Innovation.

\section{Resumen}

Objetivo: Describir las percepciones de las enfermeras gestoras sobre la relación entre Enfermería e innovación en el proceso de trabajo. Método: Se trata de un estudio cuantitativo, descriptivo, exploratorio, realizado en un hospital especializado en oncología, grande, de alta complejidad y filantrópico, ubicado en la región central de la ciudad de São Paulo. Resultados: 54 enfermeros participaron en el proceso de trabajo de administración de enfermería. Los encuestados tenían una alta percepción de la enfermería y la innovación. La representación en la innovación en enfermería está vinculada a la creación de materiales y equipos. Discusión: La innovación es un tema importante para el escenario de la salud y es parte de la gestión y el proceso de trabajo, madurado a través de la docencia, la formación continua y la experiencia. Conclusiones: La innovación, sin duda, brinda éxito al sistema y proceso de salud y, por tanto, la ausencia de un método de innovación influye en la falta de claridad en la vida cotidiana. causa poca o ninguna apreciación del tema en la práctica.

Palabras clave: Difusión de innovaciones; Practica profesional; Servicios de enfermería; Administración de enfermería; Enfermería; Innovación. 


\section{Introdução}

A campanha Nursing Now, lançada no Brasil em 2020 com o desafio Nightinhgale, que visa, em linhas gerais, incentivar o treinamento e a formação de lideranças em Enfermagem. Nesse contexto, o Mapa da Inovação é um importante projeto que se encontra alinhado com as estratégias da campanha, já que as inovações são entendidas como um caminho para aumentar a visibilidade e fortalecer a participação da categoria para a melhoria do sistema de saúde (COFEN, 2020).

Os enfermeiros são considerados profissionais inovadores, que buscam, através da sua prática, um caminho para entregar o cuidado em saúde. As tecnologias por eles desenvolvidas, como os dispositivos materiais e as ferramentas intelectuais que orientam os profissionais para a tomada de decisão em direção aos melhores resultados na assistência à saúde são provas de que a inovação está presente na categoria (Marshall, 2019).

Um estudo sobre o poder inovador da Enfermagem afirma que os enfermeiros precisam do ambiente adequado e incentivo para continuar inovando, e coloca, como reflexão, a pergunta - "O que aconteceu com todas as ideias engenhosas para melhorar o paciente?", já que as criações diárias a partir da década de 1950 passaram a ser realizadas por meio de pesquisa com métodos científicos e as ideias diária dos enfermeiros foram desvalorizadas (Marshall, 2019).

Afinal de contas, o que significa inovação? O termo em latim innovatio, definido como ato de tornar novo através de novas ideias que levam ao sucesso, pode ser entendido ainda como caminhos ainda não andados, que visa a construção de produtos, criação de tecnologias ou processos novos (ABGI, 2019)

Para Costa (2016) a inovação na saúde é compreendida como a aplicação de ideias, processos, produtos ou procedimentos que visam beneficiar a coletividade, tendo em vista o complexo contexto do cuidado ${ }^{4}$. Uma visão diferenciada do cuidado em saúde suscita o pensar em inovação e tem sido uma inquietação expressa pelas lideranças no mundo.

A Enfermagem é uma ciência e uma prática social exercida pelos enfermeiros, técnicos de enfermagem, auxiliares de enfermagem e parteiras que agrega valor ao cuidado em saúde e uma categoria profissional que mais estuda os próprios processos de trabalho (Sanna, 2007). Feldman, Ruthes, Cunha (2008) relatam que a inovação está presente nos diferentes ambientes de trabalho, mas há escassez de estudos sobre a ela quando se fala sobre a gestão dos serviços de saúde e como competência dos enfermeiros.

O processo de trabalho, por sua vez, é definido como a interação transformacional de um objeto em um determinado produto, através da ação humana, por meio de componentes como objeto, agentes, instrumentos, finalidades, métodos e produtos. Na Enfermagem são divididos em: assistir, administrar, ensinar, pesquisar e participar politicamente (Sanna, 2007).

A pergunta norteadora nesse estudo será sobre quais valores são atribuídos pelos enfermeiros que exercem a gestão nos diferentes processos de trabalho sobre a relação entre a inovação e a Enfermagem. Sendo assim, o objetivo identificar as variáveis associadas à atribuição de valor ao conceito de inovação pelos enfermeiros gestores sobre a relação da inovação e enfermagem nos processos de trabalho de um hospital oncológico.

\section{Metodologia}

Trata-se de um estudo quantitativo, descritivo, exploratório, realizado em um hospital especializado oncológico, de grande porte, de alta complexidade e filantrópico, localizado na região central da cidade de São Paulo.

Utilizou-se como critérios de inclusão os enfermeiros que desempenham papel de liderança nessa instituição, podendo ser líderes de enfermagem, supervisores de enfermagem e gerentes de operações (com atividades do tipo gestão de equipes, de recursos físicos, de materiais, de processo administrativo e gestão de indicadores assistenciais e operacionais), que aceitasse participar voluntariamente, com assinatura do Termo de Consentimento Livre e Esclarecido (TCLE). O instrumento utilizado para obtenção dos resultados da presente pesquisa foi construído pelos autores com base nos levantamentos da literatura a respeito da inovação nos contextos da saúde. 
Como critérios utilizados para exclusão foram elencadas as seguintes condições: ser enfermeiro assistencial (Júnior, Pleno ou I); ser gestor com formação acadêmica em enfermagem mas atuante nas superintendências administrativas como auditoria médica, setor financeiro e de infraestrutura; e, finalmente, o enfermeiro estar em férias e/ou licença médica no período da coleta de dados.

Os sujeitos foram selecionados após mapeamento dos processos de trabalho da enfermagem da população empregada na instituição em estudo, disponibilizado pela liderança de enfermagem. Um contato prévio com convite para participar da pesquisa foi realizado via e-mail. Após o aceite e disponibilização do TCLE, os sujeitos responderam o questionário, cujas respostam retornavam automaticamente para os pesquisadores após o completo preenchimento. A recusa de participação na pesquisa foi considerada após o insucesso de 03 (três) contatos por e-mail sem que o convidado desse retorno aos pesquisadores. O período de coleta foi entre os meses de julho e agosto de 2019.

Os dados foram tratados utilizando-se os Softwares Excel® e Statistical Package for the Social Sciences (SPSS®). Para a análise foram realizados os cálculos dedas frequências relativa e absoluta de respostas e o Teste Exato de Fisher e Teste de correção de continuidade, para avaliar associações entre as variáveis: processo de trabalho, tempo de trabalho na instituição, tempo de no cargo atual, cargo, formação gerencial e assistencial, considerando que o valor de $p<0,05$ demonstrava significância e associação.

O projeto de pesquisa foi aprovada pelos Comitês de Ética em Pesquisa da instituição proponente (a instituição de ensino que orientou a realização da investigação) e coparticipante (o hospital oncológico local do estudo), aprovados através do pareceres de $\mathrm{n}^{\circ}$ 3.337.768, CAAE 12794319.3.0000.0070 e 3.458.190, CAAE 12794319.3.3001.5432, respectivamente. Os participantes foram expostos aos procedimentos éticos, conforme prevê a Resolução $n^{\circ}$ 466/12 do Conselho Nacional de Saúde, antes de responder ao questionário.

\section{Resultados e Discussão}

Após o mapeamento realizado, o total de sujeitos elegíveis para a participação na pesquisa foi de 68 (sessenta e oito) enfermeiros gestores, sendo que 54 (cinquenta e quatro) participaram do estudo. Foram excluídos 14 (quatorze) pelos seguintes motivos: período de férias (03 enfermeiros) e licença médica (01 enfermeiro) e 10 (dez) se recusarem a participar do estudo.

A caraterização da população foi segregada em 03 (três) tipos de grupos de variáveis, sendo as características pessoais, características do processo de trabalho e características da formação, conforme pode ser visto na Tabela 1: 
Tabela 1. Caracterização da população do estudo.

\begin{tabular}{|c|c|c|c|}
\hline \multicolumn{2}{|c|}{ CARACTERÍSTICAS PESSOAIS } & $\mathbf{N}$ & $\%$ \\
\hline \multirow{2}{*}{ Sexo } & Masculino & 8 & 14,8 \\
\hline & Feminino & 46 & 85,2 \\
\hline \multicolumn{2}{|c|}{ CARACTERÍSTICAS DO PROCESSO DE TRABALHO } & $\mathbf{N}$ & $\%$ \\
\hline \multirow{5}{*}{ Processo de trabalho da enfermagem } & Administrar & 42 & 77,8 \\
\hline & Assistir & 7 & 13,0 \\
\hline & Ensinar & 2 & 3,7 \\
\hline & Participar politicamente & 2 & 3,7 \\
\hline & Pesquisar & 1 & 1,9 \\
\hline \multirow{3}{*}{ Cargo } & Supervisor & 26 & 48,1 \\
\hline & Enfermeiro líder & 25 & 46,3 \\
\hline & Gerente de Unidade de Negócio & 3 & 5,6 \\
\hline \multirow{6}{*}{$\begin{array}{l}\text { Tempo de trabalho na } \\
\text { instituição pesquisada }\end{array}$} & 0 a 5 anos & 4 & 7,4 \\
\hline & 6 a 10 anos & 19 & 35,2 \\
\hline & 11 a 15 anos & 18 & 33,3 \\
\hline & 16 a 20 anos & 7 & 13,0 \\
\hline & 21 a 25 anos & 4 & 7,4 \\
\hline & 26 a 30 anos & 2 & 3,7 \\
\hline \multirow{4}{*}{$\begin{array}{c}\text { Tempo no } \\
\text { carga atual }\end{array}$} & 0 a 5 anos & 33 & 61,1 \\
\hline & 6 a 10 anos & 15 & \\
\hline & 11 a 15 anos & 4 & 7,4 \\
\hline & 16 a 20 anos & 2 & 3,7 \\
\hline \multicolumn{2}{|c|}{ CARACTERÍSTICAS DA FORMAÇÃO } & $\mathbf{N}$ & $\%$ \\
\hline \multirow{4}{*}{ Última formação } & Especialização & 24 & 44,4 \\
\hline & MBA & 23 & 42,6 \\
\hline & Mestrado & 6 & 11,1 \\
\hline & Doutorado & 1 & 1,9 \\
\hline \multirow{2}{*}{ Formação assistencial em oncologia } & Não & 25 & 46,3 \\
\hline & Sim & 29 & 53,7 \\
\hline \multirow{2}{*}{ Formação na área da gestão } & Não & 17 & 31,5 \\
\hline & Sim & 37 & 68,5 \\
\hline
\end{tabular}

Fonte: Autores.

Para a caraterização da população foi possível segregar em 03 (três) tipos de grupos de variáveis (características pessoais, características do processo de trabalho e características da formação). Majoritariamente participou da presente pesquisa enfermeiras, pertencentes ao processo de trabalho da enfermagem "administrar", com cargo Supervisor de Enfermagem, com tempo de casa entre 06 a 10 anos, entre 0 a 5 anos de experiência no cargo atual, especialista, com formação na assistencial da oncologia e gestão.

Na Tabela 2 se pode ver as respostas dos sujeitos à afirmação "Na sua visão, a Enfermagem é...” e obtive-se: 
Tabela 2. Percepção sobre a inovação, a enfermagem e eficiência - São Paulo, 2019.

\begin{tabular}{|c|c|c|}
\hline NA SUA VISÃO, A ENFERMAGEM É... & FREQUÊNCIA & \% \\
\hline Inovadora e eficiente & 32 & 59,3 \\
\hline Inovadora, mas não tão eficiente & 9 & 16,7 \\
\hline Não inovadora, mas eficiente & 10 & 5,6 \\
\hline Nenhuma das opções & 3 & $100 \%$ \\
\hline Total & 54 & \\
\hline
\end{tabular}

Fonte: Autores.

A pergunta 1 foi construída utilizando o conceito abordado por Casimiro Cavaco Dias (2015) para categorizar os hospitais europeus quanto ao grau de inovação, podendo ser baixo ou alto. No estudo, o autor utilizou a classificação as seguintes opções: "Inovadora e eficiente", "Inovadora, mas não tão eficiente", "Não inovadora, mas eficiente" ou "Nem inovadora e nem eficiente" e concluiu que existe uma positiva e importante correlação entre inovação e desempenho organizacional. A minoria dos hospitais (9\%) foi considerada "inovador e eficiente" e essa avaliação se deu pelos seguintes motivos: aproveitamento do potencial da inovação, pelas garantias das melhorias de desempenho, por ser hospital especializado, pelo porte de leitos (grande porte), nível elevado de flexibilidade organizacional, utilização de novas tecnologias e de cooperação externa.

Os enfermeiros também foram convidados a descrever a sua percepção sobre a Enfermagem e a inovação. Majoritariamente, 59,3\% dos enfermeiros concordam que a Enfermagem é "inovadora e eficiente".

A influência da instituição em que os enfermeiros entrevistados estão inseridos pode ter auxiliado, já que as características organizacionais (hospital especializado, de grande porte, com elevada utilização de novas tecnologias e com um robusto desenvolvimento organizacional) favorecem para esta percepção. Para essa questão, foram utilizados testes estatísticos, conforme descrito no método e, mas não houve associações entre as variáveis (Dias, 2015).

Os enfermeiros são desafiados a construírem uma cultura inovadora capaz de melhorar a entrega do cuidado, no entanto, ainda são observadas lacunas significativas nas competências de inovação por parte dos enfermeiros e por isso treinamentos sobre o tema já que pouco se sabe sobre da aplicação prática da inovação no contexto da prático e educacional (Joseph, Huber \& Bair, 2019).

É por meio da inovação que os enfermeiros poderão contribuir com a organização no desempenho eficaz do trabalho e criar melhorias sustentáveis (Joseph, Huber, Bair, 2019). A American Organization of Nurse Executives (2020) destaca que as instituições de saúde precisam funcionar de forma inovadora e, para isso, é preciso estimular os profissionais, apoiando de forma contínua o aprendizado, estimulando o pensamento crítico e o trabalho em equipe.

Um estudo sobre os níveis de inovação entre enfermeiros líderes apontou que os gestores apresentam comportamento significativamente melhor para o processo de inovação. O mesmo estudo destacou que para desenvolver o clima para a inovação, o estilo da liderança e competência são elementos críticos, já que liderar e inovar são vitais para o processo de mudança e melhoria (Stilgenbauer, Fitzpatrick, 2019).

Existem relatos de enfermeiros gestores de que a inovação é a união de um trabalho em equipe, construção da capacidade da liderança e capacidade de interação em todos os níveis. Eles discutem que o enfermeiro líder precisa preparar o ambiente, educar as equipes para o processo de mudança, comunicar sobre o valor que a inovação traz, já que, sem o apoio do líder, dos mais diferenciados níveis, incluindo a alta gerência, é muito difícil inovar (Clement-O’brien, Polit, Fitzpatrick, 2011). 
Em um estudo brasileiro sobre liderança de enfermeiros em Unidade de Terapia Intensiva discutiu-se que liderar centralizando a atenção nas relações humanas, levando em consideração a confiança nas relações, incentivando à criatividade e inovação, contribui para o aumento da produtividade e melhora dos resultados no trabalho (Conz et al, 2019).

Para essa questão foram aplicados os testes estatísticos (Exato de Fisher, Teste de correção de continuidade e Quiquadrado) que não mostram associações entre as variáveis pesquisadas.

No Gráfico 1, se pode ver a incidência de reconhecimento de inovações trazidas pela e Enfermagem pelos participantes do estudo.

Gráfico 1. Percepção dos exemplos das inovações na Enfermagem - São Paulo, 2019.

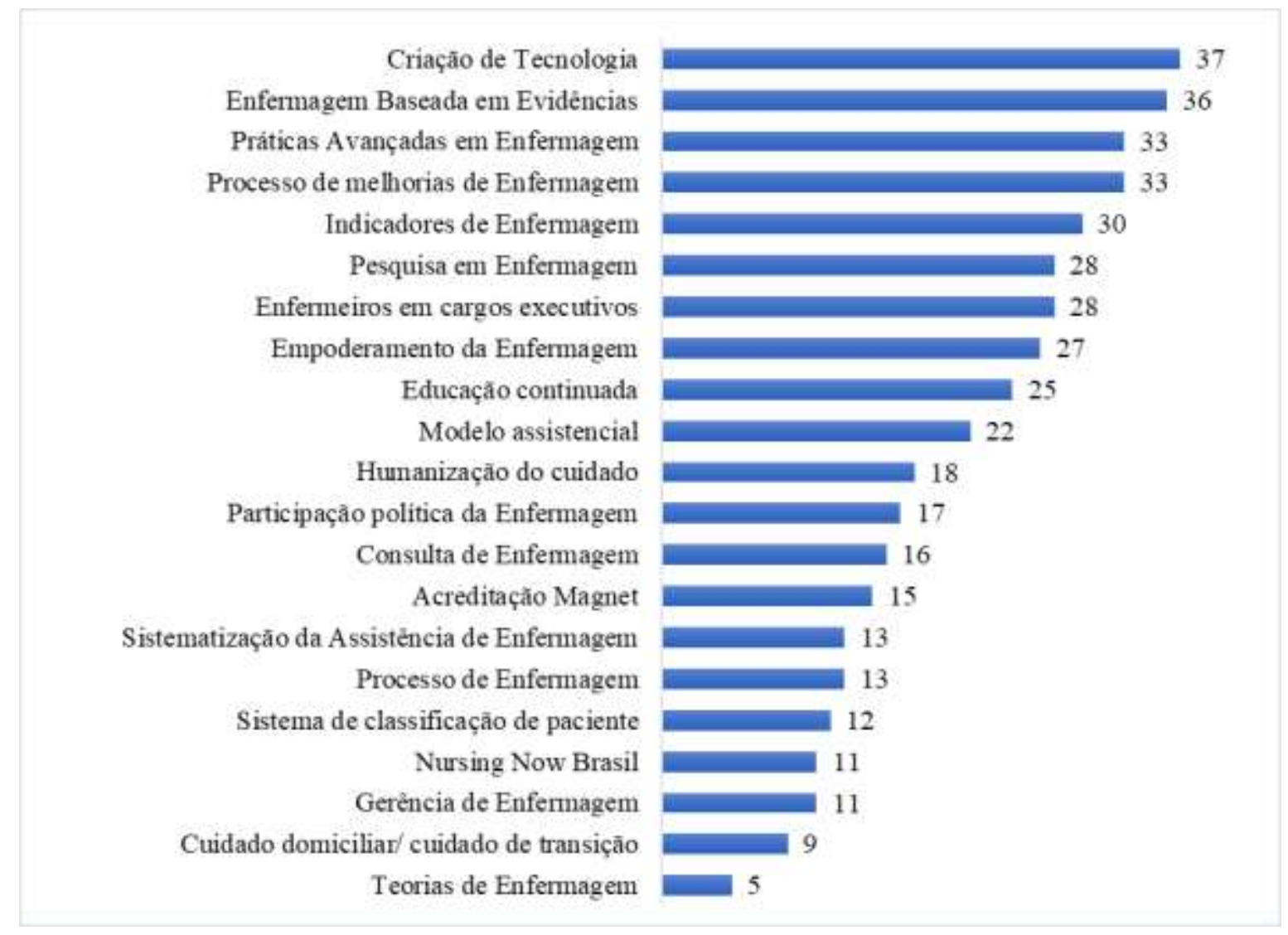

Fonte: Autores.

No Gráfico 2, são apresentados os resultados sobre as barreiras que atrapalham a enfermagem para inovar apontadas pelos respondentes do questionário 
Gráfico 2. Frequência da percepção sobre as barreiras que atrapalham a inovação - São Paulo, 2019.

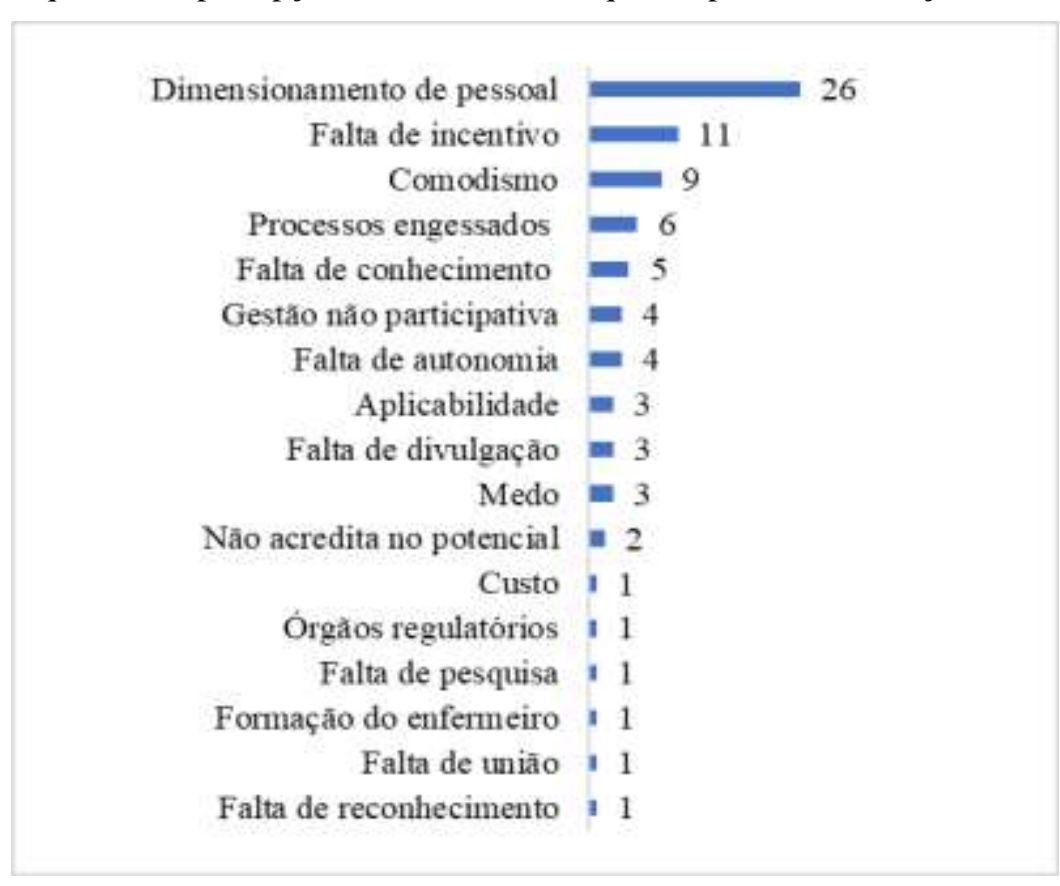

Fonte: Autores.

No Gráfico 1, os enfermeiros participantes da pesquisa foram expostos a uma listagem de exemplos de, desde inovações mais antiga até as mais atuais. Para essa análise é importante considerou-se os conceitos de tecnologias leve, levesdura e dura.

Segundo as percepções dos enfermeiros sobre inovações desenvolvidas como parte do corpo de conhecimento da enfermagem a criação tecnológica (criação de maquinários, equipamentos, coberturas) foi considerada majoritariamente com a tradução da inovação da Enfermagem, enquanto as teorias de Enfermagem foram consideradas, em minoria.

A maioria dos exemplos apresentados aos respondentes são de inovação com tecnologia leve e leve-dura. A tecnologia dura é caracterizada pela utilização dos instrumentos, normas e equipamentos tecnológicos, as leves-dura são os saberes estruturas (normas, protocolos e conhecimento) e a tecnologias leves são utilizadas nas relações humanas, (na produção de vínculo, autonomização, acolhimento, na conexão interpessoal entre profissional e usuário) (Salvador, 2012; Sabino, 2016).

As teorias de enfermagem são um tipo de tecnologia leve-dura e um referencial teórico para a criação de qualquer tipo de inovação. Ao criar um material ou equipamento que o próprio paciente manipule, efetivamente, diretamente há aplicação de uma teoria de enfermagem. Fica nítido que a teoria, por ser uma tecnologia leve, passa a não ser entendida como uma inovação.

Diante dos presentes resultados, é possível considerar a importância de pensar mais sobre a inovação na Enfermagem, pois a utilização de tecnologias leve e leve-dura não faz a Enfermagem menos inovadora que outras ciências, que criam equipamentos e medicamentos, por exemplo. O uso de todas essas formas de tecnologias é importante de igual forma para o processo de inovação.

Estudos sobre tecnologia e inovação para o cuidado de enfermagem destacam que inovações tecnológicas em saúde não devem ser vistas apenas com a implantação de um produto, mas deve levar em consideração o processo de construção do conhecimento e instrumento que se ligam para determinar uma forma de cuidar (Salvador, 2012; Cestari et al, 2017).

Um importante momento vivenciado mundialmente é a campanha Nursing Now, que recomenda que governos e 
agências internacionais busquem a valorização, a criação de políticas de formação e desenvolvimento da Enfermagem, com metas que visam a melhoria das condições de trabalho e remuneração, o aumento do número de enfermeiros em posição de liderança em direção da disseminação das práticas de Enfermagem efetivas e inovadoras, já que o sistema de saúde depende desta e para que haja sucesso com esses objetivos, os enfermeiros precisarão utilizar a inovação para auxiliar em cada etapa desse processo (Crisp, Iro, 2019; Nursing Now Brasil, 2016; Salvage, 2019).

As novas ideias devem se sobressair à confiança da sabedoria convencional, aos anos de experiência e a agilidade que são as características que determinarão o enfermeiro do futuro, já que o mundo contemporâneo exige profissionais capazes de utilizar métodos modernos e soluções diferentes (Munhoz, Ramos, Cunho, 2008; Sanford, Janney 2019).

E dentre as barreiras que dificultam ação de inovar da Enfermagem figurou o dimensionamento de pessoal. Assim como em outras pesquisas, essa condição apresentou-se como a maior barreira para a prática da enfermagem. Considerando-se que a pesquisa foi respondida por líderes de enfermagem e que o dimensionamento de pessoal é um problema de grande desafio na área da gestão, pode-se entender a expressividade numérica que lhe foi atribuída.

Outras barreiras, as quais têm relação com as características pessoais dos profissionais, como "comodismo", "falta de conhecimento", "não acredita no potencial", "medo", "formação do enfermeiro", entre outras, secundaram a lista das dificuldades para inovação na Enfermagem indicadas pelos respondentes. Estudos consideram que as características pessoais têm um efeito direto e de significância para o processo de inovação, como principal impulsionadores da mudança e ajudam na difusão mais rápida da qualidade do cuidado ao paciente (Munhoz, Ramos, Cunho, 2008; Clement-O’brien,, Polit, Fitzpatrick, 2011).

Enfermeiros entrevistados sobre as inovações percebidas em um centro médico acreditado pelo selo Magnet ${ }^{\circledR}$, em Iowa, nos Estados Unidos pontuaram "comunicação interna", "espaço e equipe" "laboratório e exames necessários", "seguro de cobertura de saúde", "falta de força base de evidências científicas", "conhecimentos e atitudes" como sendo barreiras para inovação. A inovação faz parte do desenvolvimento de uma cultura e por isso leva tempo, mas deve ser percebida como um valor, pois assim é possível ganhar apoio do pessoal interno da instituição para adotar mudança na prática (Clement-O’brien,, Polit, Fitzpatrick, 2011).

\section{Considerações Finais}

O estudo sobre as percepções atribuídas pelos enfermeiros de um hospital oncológico sobre inovação e a identificação relação entre a inovação e a Enfermagem é importante, pois promovem o aumento de conhecimento e a capacidade da enfermagem nos ambientes de atuação da Enfermagem independentemente do tipo de tecnologia aplicada.

A ausência de um método de inovação influencia a falta de clareza no dia a dia, que provoca a baixa ou não valorização do tema, conforme discutido acima. Desse modo, a inovação deve ser tratada e entendida como uma política e com metodologia definida, visto que tem sido um valor defendido no planejamento estratégico de muitas instituições.

A indisponibilidade de estudos a respeito de outras percepções de enfermeiros sobre a inovação no cenário brasileiro

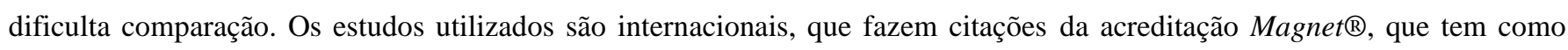
premissa a difusão de inovação entre a e Enfermagem e, mas ainda não é uma realidade brasileira. O cenário do estudo (hospital grande, especializado, com valor de inovação definido) favoreceu a percepção coletada no presente estudo, conforme descrito em literatura utilizada no presente estudo.

A inovação, sem sombra de dúvida, proporciona o sucesso ao processo. O sistema de saúde necessita da inovação. A Enfermagem necessita da inovação. O cuidado em saúde necessita da inovação, ou seja, a inovação é um caminho pelo qual buscaremos a sustentação do sistema de saúde, sendo possível agregar valor aos usuários e profissionais dos serviços de saúde. Por sim, sem dúvidas, a Enfermagem é uma inovadora e eficiente. Destaca-se a necessidade de novas pesquisas relacionando 
estas e outras variáveis para trabalhos futuros na intenção de aperfeiçoar o conhecimento da inovação pela enfermagem e novas descobertas e influências destas para a ciências.

\section{Agradecimentos}

A liderança de enfermagem que participou gentilmente da realização do presente estudo e trouxeram contribuições importantes para a construção do conhecimento da enfermagem.

\section{Referências}

ABGI Accelerating innovation (2019). Inovação: o que é? <http://brasil.abgi-group.com/>.

American Organization of Nurse Executives. (2016). The Innovation Road Map: A Guide for Nurse Leaders. <https://www.nursingworld.org/globalassets/ana/innovations-roadmap-english.pdf>.

Cestari, V.R.F. et al (2017). Aplicabilidade de inovações e tecnologias assistenciais para a segurança do paciente: revisão integrativa. Cogitare Enferm. 22(3). e45480.

Clement-O'brien, K., Polit, D. F., \& Fitzpatrick, J. J. (2011). Innovativeness of nurse leaders. J Nurs Manag. 19(4), $431-438$.

Conselho Federal de Enfermagem (2019). Nursing Now quer formar enfermeiros líderes e ampliar mapa de inovações. <http://www.cofen.gov.br/nursingnow-quer-formar-enfermeiros-lideres-e-ampliar-mapa-de-inovacoes_75531.html>.

Conz, C.A. et al (2019). Atuação de enfermeiros líderes de Unidade de Terapia Intensiva: Abordagem Compreensiva. Enfermagem em Foco, 10 (4): 41 -46.

Costa, L.S. (2016). Inovação nos serviços de saúde: apontamentos sobre os limites do conhecimento. Cad. Saúde Pública, $32(2)$, 1-12.

Crisp, N \& Iro, E. (2018). Nursing now campaign: raising the status of nurses. The Lancet, (10124):920-1.

Dias, C.C. (2015). O valor da inovação: criar o futuro do sistema de saúde. Almedina.

Feldman, L. B., Ruthes, R. M., \& Cunha, I. C. K. O. (2008). Criatividade e inovação: competências na gestão de enfermagem. Revista Brasileira Enfermagem. 61(2): $239-42$.

Joseph, M. L., Huber, D. L., \& Bair, H. A. (2019). Typology of Innovations in Nursing. JONA. 49(7/8).

Marshall, D. R. (2019). Unleashing the Power of Nurse Innovators. JONA. 49(2), 55-56.

Munhoz, S., \& Ramos, L. H \& Cunha, S. C. K. O. (2008). Eficiência e eficácia do desempenho da enfermagem em procedimentos técnicos. Rev. Bras. Enferm. $61(1)$.

Nursing Now Brasil. (2016). COFEN disponibiliza material da campanha Nursing Now. <http://nursingnowbr.org/noticia/cofen-disponibiliza-material-dacampanha-nursing-now>.

Pereira A. S. et al. (2018). Metodologia da pesquisa científica.

Sabino, L. M. M. et al (2016). Uso de tecnologia leve-dura nas práticas de enfermagem: análise de conceito. Aquichan. 16(2), 230-239.

Salvador, P. T. C. O. et al (2012). Tecnologia e inovação para o cuidado de Enfermagem. Rev. Enferm. UERJ, $20(1): 111$.

Salvage, J. (2018) Uma nova história da enfermagem. Revista de Enfermagem Referência. 17, 2-10.

Sanford, K., \& Janney, M. (2019). Preparing the Nurse Executive of the Future. JONA. 49(4).

Sanna, M. C. (2007). Os processos de trabalho em Enfermagem. Revista Brasileira de Enfermagem, 60(2), 221-4.

Stilgenbauer, D. J., \& Fitzpatrick, J. J. (2019). Levels of Innovativeness Among Nurse Leaders in Acute Care Hospitals. JONA. 49(3), 150-155. 